\title{
1 EL DISEÑO Y EL DESARROLLO CURRICULAR EN LA EDUCACIÓN SECUNDARIA OBLIGATORIA
}

\author{
José Luis Rodríguez Diéguez
}

Catedrático de Didáctica

Universidad de Salamanca

retendo con este trabajo presentar una perspectiva del análisis del currículo que se llevó a cabo en el INCE durante el año 1998, coordinado por el profesor García Garrido, y en el que me responsabilicé del estudio del diseño y desarrollo curricular.

Intento conceder un relieve mayor a las aportaciones cualitativas que efectuaron los profesores que colaboraron en este trabajo que a los datos estrictamente cuantitativos procedentes de la encuesta, como luego se verá.

\section{EL ORIGEN DE ESTE ESTUDIO}

Los resultados de este estudio proceden del Diagnóstico del Sistema Educativo. La escuela secundaria obligatoria, 1997, llevado a cabo por el Instituto Nacional de Calidad y Evaluación, en respuesta a la actualización del Proyecto Global del Plạn de actuación 1994-97.

En este Proyecto Global se definía el siguiente objetivo:

Indagar la situación en la que se encuentra el sistema educativo español y su grado de eficiencia real, en el momento en el que las competencias educativas van a ser transferidas a todas las Comunidades Autónomas. 
Uno de los cinco campos con los que se pretendía dar respuesta a este objetivo inicial aparecía formulado así:

Qué y cómo se enseña a los alumnos (Evaluación de los planes de estudio y de los métodos de enseñanza).

Se trataba de estudiar si los planes de estudio vigentes resultan los más adecuados a las necesidades de los alumnos y de la sociedad española en su conjunto.

Una de las cinco Comisiones Especializadas, la número 2, se encargó de llevar a cabo los planteamientos y las actuaciones orientadas a detectar esta situación en la Educación Secundaria Obligatoria.

El equipo investigador estuvo constituido por Rosario Beltrán de Tena (Universidad de Salamanca), Jaime García-Gallo Pinto (INCE), María Jesús Pérez Zorrilla (INCE), María José Rodríguez Conde (Universidad de Salamanca) y Serafín Sánchez Sánchez (Inspector de Educación de Salamanca).

Se acordó por la Comisión correspondiente llevar a cabo el estudio, considerando como fuente primordial de información a los profesores de Educación Secundaria Obligatoria. Al margen del valor que se concede habitualmente a la evaluación interna de los currículos, en este caso pesaron también razones de tiempo.

Para ello se determinó iniciar el trabajo mediante una doble perspectiva: una encuesta al profesorado del nivel correspondiente, y una serie de reuniones de lo que se denominaron "Grupos de Diagnóstico", cuyo sentido principal era el de proporcionar información directa y verbal sobre las opiniones de profesores y profesionales vinculados al nivel de enseñanza que se estudiaba.

\section{METODOLOGÍA}

\subsection{LA ENCUESTA}

Después de un proceso de documentación y debate, que ocupó una amplia serie de sesiones a la Comisión en pleno, se llegó a la definición de una encuesta que constituiría una de las fuentes informativas para nuestro estudio.

Constaba de 325 preguntas formuladas sobre un conjunto de 47 cuestiones. Las respuestas -en su inmensa mayoría- exigían una valoración en una escala de 1 a 5. 
A continuación se muestran, como ejemplo, dos de las cuestiones formuladas en la encuesta:

En la cuestión 18 se preguntaba sobre los instrumentos que el profesor utiliza para llevar a cabo la programación de su trabajo. Este es el enunciado:

¿Con qué frecuencia utiliza Vd. los siguientes instrumentos o documentos para programar lo que va a hacer en clase?

a) El proyecto educativo de Centro

b) El proyecto curricular de etapa/Centro

c) La programación general anual de Centro

d) Las programaciones didácticas de departamento

e) La programación de aula

f) Las notas y apuntes personales de cursos pasados

g) El libro de texto

h) La guía didáctica o la programación que acompaña al libro de texto

i) El Decreto de currículo de Educación Secundaria Obligatoria

j) Los objetivos que pretendo conseguir con el alumnado

k) Los materiales y recursos de enseñanza que tengo a mi alcance

Con relación a las actividades que se realizan en el aula, uno de los conjuntos de preguntas era este:

29. Indique con qué frecuencia ha empleado, durante el mes anterior a la contestación de este cuestionario, los siguientes procedimientos con sus alumnos de Educación Secundaria Obligatoria:

a) Trabajo individual del alumnado en el aula

b) Trabajo en grupo del alumnado dirigido por el profesor

c) Trabajo en equipo por pequeños grupos y posterior exposicion por el alumnado de las conclusiones o síntesis del trabajo realizado

d) Trabajos de investigación y descubrimiento en equipo de toda la clase

e) Tareas para realizar en.casa

f) Explicación general del profesor a toda la clase, sin participación del alumnado

g) Explicación del profesor con intervenciones y preguntas del alumnado

h) Revisión en grupo de las tareas realizadas por el alumnado en clase 
i) Exposición de un tema por parte de algún o algunos alumnos

j) Visionado de un video

k) Visionado de diapositivas

l) Debate de un tema con toda la clase

m) Corrección de las actividades o tareas realizadas en casa

La población objeto del estudio es el conjunto de profesores que durante el curso 1996/97 impartía clases a alumnos de la ESO en alguna de las siguientes cinco materias: Lengua, Matemáticas, Ciencias Sociales, Ciencias Naturales y Tecnología.

Por diversas razones se excluyeron los ámbitos territoriales de Andalucía, Canarias, Ceuta y Melilla.

La muestra total estuvo compuesta por 3287 profesores, respondiendo a la siguiente distribución muestral:

\begin{tabular}{|l|c|r|c|c|r|c|}
\hline & Población & $\%$ & $\begin{array}{c}\text { Muestra } \\
\text { diseñada }\end{array}$ & $\begin{array}{c}\text { Muestra } \\
\text { lograda }\end{array}$ & $\%$ & Peso \\
\hline Cataluña & 28.448 & 19,42 & 500 & 458 & 91,6 & 1,56 \\
Galicia* $^{*}$ & 13.431 & 9,17 & 500 & 542 & 108,4 & 0,62 \\
Navarra** $^{\star *}$ & 3.495 & 2,39 & 500 & 427 & 85,4 & 0,17 \\
Valencia* $^{*}$ & 17.128 & 11,69 & 500 & 528 & 105,6 & 0,80 \\
$\begin{array}{l}\text { País Vasco } \\
\text { Territorio } \\
\text { MEC }\end{array}$ & 10.450 & 7,13 & 500 & 441 & 88,2 & 0,49 \\
TOTAL & 73.531 & 50,20 & 1.000 & 891 & 89,1 & 1,71 \\
\hline
\end{tabular}

*Estas comunidades ampliaron su muestra con la contestación de un cuestionario más, contestado por el profesorado que imparte la lengua propia de la comunidad.

** los 427 profesores de Navarra se corresponden con la población que imparte ESO en la comunidad en el curso 96-97

\subsection{LOS "GRUPOS DE DIAGNÓSTICO”}

Los datos de la encuesta se pretendían armonizar con los procedentes de las sesiones de los Grupos de Diagnóstico.

Los Grupos de Diagnóstico, como ya he publicado en alguna otra ocasión (Rodríguez Diéguez, 1998) son una variante de la técnica de "entrevista orientada", que se iniciara con Merton en los años cuarenta (1946) y que estructuraron después el mismo Merton, Fiske y Kendall (1956) Inicialmente estaba destinada al estudio de mensajes de los medios de comunicación de masas. La técnica originaria de Merton suele denominarse ahora "focus group". 
Un Grupo de Diagnóstico es una sesión de trabajo de un grupo de personas, constituido por un número no excesivamente amplio de especialistas o conocedores de un tópico, y un coordinador que establece y orienta un intercambio de informaciones y opiniones sobre unos temas previamente acotados y establecidos.

Un Grupo de Diagnóstico tiene las siguientes características:

Se trata de una variedad de entrevista, de un cuestionario verbal al servicio del objetivo marcado en la investigación. El investigador busca información cualificada sobre el tema que estudia. Esta entrevista -0 "tertulia", como la denominó alguno de los participantes- se realiza con un grupo de personas de características previamente definidas de modo general. La característica básica viene dada por el conocimiento del tema que se aborda en la reunión.

Es necesaria la presencia de un moderador que gestione su desarrollo. El moderador debe estar entrenado en la técnica y/o en el tema del que se pretende obtener información.

Deben registrarse las aportaciones de los participantes a fin de facilitar el posterior análisis de las ideas aportadas.

En las sesiones de los Grupos de Diagnóstico se plantearon una serie de problemas con la intención de que afloraran las opiniones de los participantes sobre aspectos tales como:

Las tareas, funciones e instrumentos necesarios y utilizados para la preparación de las clases.

Los recursos y los medios didácticos de los que se hace uso con mayor frecuencia.

Los problemas derivados de la integración, la diversificación y las adaptaciones curriculares.

El clima del centro: tono, sentido y funcionalidad de las relaciones del profesor con alumnos, compañeros, padres.

Las funciones de la Comisión de Coordinación Pedagógica y su posible eficacia.

Las necesidades más urgentes a las que habría que dar respuesta para incrementar la calidad de la enseñanza en la ESO...

Se comenzaba la sesión pidiendo a cada uno de los participantes su visión global sobre la Educación Secundaria Obligatoria. Se solicitaba sobre este punto una intervención breve, de unos cinco minutos de duración, que podría dar lugar un debate inmediatamente posterior, previo al listado que se ha visto. 


\subsection{EL TRATAMIENTO DE LOS DATOS}

A fin de tratar de un modo más homogéneo los resultados de la encuesta se trataron los datos mediante un análisis factorial de segundo orden. Las características de la Comisión Especializada que generó la encuesta exigió en bastantes casos una negociación de las formulaciones, las preguntas y su sentido, que en algunas ocasiones derivó en planteamientos redundantes. De aquí que el análisis de segundo orden garantizara una mayor capacidad de síntesis de los resultados.

Para llegar a ello se realizaron una serie de agrupamientos de las variables, que al final permitieron considerar cuatro bloques conceptuales:

1. El diseño curricular.

2. El desarrollo curricular

3. Los componentes contextuales del currículo.

4. Las culturas de la Educación Secundaria Obligatoria.

En este estudio se van a presentar los resultados de los dos primeros apartados.

Las casi setenta horas de grabación obtenidas en las sesiones de los Grupos de Diagnóstico, tras su análisis y codificación, proporcionaron una base de datos con un total de 6727 registros, que se utilizaron para describir situaciones no previstas inicialmente en la encuesta y para confirmar y matizar los resultados obtenidos.

\section{LOS RESULTADOS}

Estudiar los planes de estudio y los métodos de enseñanza supone realizar una evaluación del currículo, una evaluación de lo que se proyecta enseñar y cómo se pretende hacerlo, el diseño curricular, y de la puesta en práctica de lo proyectado, el desarrollo curricular.

La diferenciación entre estos dos procesos queda suficientemente explícito en cualquiera de los volúmenes del "Diseño Curricular Base".

Las intenciones y el plan de acción que se establecen en el curriculo se plasman, en último término, en una determinada práctica pedagógica. El currículo incluye tanto el proyecto como su puesta en práctica. Esta propuesta curricular diferencia las dos fases, reservando el término de Diseño del currículo para el proyecto que recoge las 
intenciones y el plan de acción, y el de Desarrollo del currículo para el proceso de puesta en práctica. Estas dos fases tienen una enorme relación entre sí, ya que la información obtenida de la aplicación del currículo debe servir para ir modificando el diseño, ajustándolo progresivamente a las condiciones reales. Sólo llevando a cabo este ciclo completo se respeta la naturaleza dinámica del curriculo, evitando que se convierta en una serie de principios fosilizados incapaces de generar ningún tipo de innovación educativa. (Ministerio de Educación y Ciencia, 1989).

Los componentes complementarios que se han señalado más atrás no se abordan, pese a su evidente interés, en este artículo.

\subsection{EL DISEÑO CURRICULAR EN LA EDUCACIÓN SECUNDARIA OBLIGATORIA}

La reforma educativa tuvo como elemento inicialmente emblemático la difusión terminológica en nuestro país del ambiguo concepto de currículum, de pura raigambre latina, pero introducido a partir de la cultura escolar anglosajona.

En los DCB ya citados se define el currículum así:

En la actualidad la oferta y las oportunidades educativas se compendian en la noción de currículo que abarca todo aquello que el medio escolar ofrece al alumno como posibilidad de aprender: no solo conceptos, sino también principios, procedimientos y actitudes; y que abarca, además, tanto los medios a través de los cuales la escuela proporciona esas oportunidades, cuanto aquellos por los que evalúa los procesos mismos de enseñanza y aprendizaje. (Ministerio de Educación y Ciencia, 1989).

Lo que el profesor tiene que hacer para preparar sus clases, de acuerdo con la reforma, es un diseño curricular. Esta reforma es la de los diseños curriculares, como la de la Ley General de Educación fue, en principio, la reforma de las fichas, o la de 1965 la de las Unidades Didácticas. Todo ello pese a que la definición legislativa de currículo pretende una clarificación de conceptos frente a la imprecisión inicial ya mencionada. La LOGSE, por ejemplo, en su artículo 4.1 estipula de este modo el concepto de currículum:

A los efectos de lo dispuesto en esta ley, se entiende por curriculo el conjunto de objetivos, contenidos, métodos pedagógicos y criterios de evaluación de cada uno de los niveles, etapas, ciclos, grados y modalidades del sistema educativo que regularán la práctica docente. 
Los objetivos constituyen uno de los elementos esenciales del currículo y, por tanto, de su diseño.

Los objetivos de la enseñanza pueden definirse como la formulación de las modificaciones de comportamientos y capacidades que deben alcanzar los alumnos como consecuencia de un determinado proceso de enseñanza y aprendizaje.

El objetivo tiene una perspectiva esencialmente pronóstica, por cuanto suele formularse en una dimensión hipotética (el alumno habrá de conocer..., será capaz de realizar ..., debería valorar...). Se trata de las intenciones a las que se pretende llegar por medio de un ciclo, un curso o un programa de enseñanza.

Las distintas áreas estructuran sus contenidos en tres apartados básicos: contenidos conceptuales, procedimentales y actitudinales.

Es necesario, sin embargo, considerar también la dimensión diagnóstica del objetivo. La determinación de la consecución de los objetivos constituye la esencia de los problemas de la evaluación. Esta relación prácticamente indisoluble entre objetivos y evaluación es una constante aceptada, hasta tal punto que uno de los componentes tradicionales de cierto tipo de objetivos -los criterios de evaluación- han sido, en el caso de la Reforma Educativa de la LOGSE, considerados como componente específico de la evaluación. La precisión del tipo de capacidad que habrá de poner en ejercicio el alumno para manifestar su dominio del objetivo, necesidad sentida e incluso exigida por el profesorado, se convierte así en elemento del proceso de evaluación. Objetivos y criterios de evaluación se consideran elementos básicos del currículo en la caracterización de la LOGSE.

Los objetivos de la enseñanza, las intenciones educativas se explicitan y configuran en unas áreas, en sustitución de las tradicionales asignaturas, de las se distinguen tres categorías diferentes:

Áreas curriculares, que son aquellos "agrupamientos de los contenidos en conjuntos coherentes en torno a disciplinas afines".

Temas transversales, que se caracterizan como "grandes temas que engloban múltiples contenidos que difícilmente pueden adscribirse específicamente a ninguna de las áreas.

Asignaturas opcionales, que se entienden como la vía de flexibilización del currículo, y que se concretan en una serie de materias y áreas que el alumno puede elegir.

Las distintas áreas estructuran sus contenidos en tres apartados básicos: contenidos conceptuales, procedimentales y actitudinales. 
La determinación de los objetivos constituye uno de los elementos básicos del currículum, así como una tarea a llevar a cabo normalmente en el diseño curricular.

La definición de objetivos en el ámbito escolar se lleva a cabo a través de un proceso de concreción progresiva, a partir de documentos legales de la máxima generalidad, y se va perfilando en otros textos, legales o técnicos, de modo más o menos coherente.

La necesidad de realizar una serie de funciones anticipadoras de la ejecución del trabajo es patente en casi todas las actividades humanas. En ámbitos distantes de la enseñanza se suele denominar a esta función planificación o programación. Ambos términos se han utilizado habitualmente en la literatura pedagógica. Ahora se denomina diseño curricular.

El diseño curricular aparece expresado con niveles distintos de concreción vinculados de alguna manera a los niveles de precisión de los objetivos. La propuesta dominante hace referencia a tres niveles sucesivos de especificación: el Proyecto Curricular de Centro, el Proyecto Curricular de Etapa o Departamento y la Programación de Aula.

El diseño curricular oficial presenta siempre una serie de limitaciones como consecuencia de su homogeneidad, por mucho que se pretenda un currículo abierto y flexible.

Esa perspectiva homogeneizadora se ve contrapuesta a la situación de la enseñanza en cada entorno concreto. Se impone una adaptación curricular al ambiente. La programación siempre ha insistido en la exigencia de localismo. Es aquí donde se ha de efectuar una primera inflexión de los contenidos y objetivos. No tiene el mismo sentido el estudio del proceso de elaboración del pan en un pueblo de la meseta castellana que en una gran urbe industrial. Esa exigible adaptación es la que, en función de esta definición de los niveles, permite la consideración de las características diferenciales de cada centro. Es el margen de autonomía curricular que se concede a cada profesor o a cada centro.

El tercer nivel que se indica en este caso es la programación de aula, que presupone ya la adaptación no sólo al entorno social general de la escuela, sino también a la situación y condicionantes de la misma.

\subsubsection{La valoración de las demandas de la Administración}

De lo analizado hasta aquí, el parentesco, si no la similitud entre el sentido de "currículum" y el de programa o programación es evidente, y hace que la reforma no suponga, en ciertos casos y a ojos de los profesores, nada más que una pura reforma terminológica. 
Uno de los participantes en las sesiones de los Grupos de Diagnóstico dice que:

... a todos nos ha pasado un poco lo mismo cuando hemos empezado con la Reforma, el lenguaje de la Reforma: proyecto curricular, niveles de concreción, etc. Todo eso nos ha pillado como diciendo: bueno, ¿y esto, realmente, qué es?. Luego, cuando realmente te pones a leer, bueno, pues ves que es un poco más de lo que había antes, sólo que con otro tipo de terminología. (LEProf 21).

No se trata de una opinión perdida entre las más de seis mil con las que se ha trabajado en la base de datos procedente de los Grupos de Diagnóstico. Es un planteamiento relativamente frecuente en las informaciones que se han manejado.

La preparación de la actividad del aula, tal como se regula en la Reforma educativa, supone que para la planificación del proceso educativo los miembros de la comunidad escolar, fundamentalmente los profesores, han de tomar una serie de decisiones compartidas y cooperativas que habrán de plasmarse en los tres documentos básicos ya señalados: el Proyecto Educativo de Centro, el Proyecto Curricular de Etapa y la Programación General del Centro.

Por medio de estos instrumentos se pretendía sustituir las programaciones anteriores, por considerar que tenían un sentido predominantemente disfuncional y burocrático.

La valoración que hacen los profesores en la encuesta sobre los objetivos, las áreas y los temas transversales es muy alta, se sitúa entre la valoración de "bastante importante" y "muy importante". No se plantean discrepancias significativas ni en los datos recogidos en la encuesta ni en los "grupos de diagnóstico":

Yo creo que los objetivos que están planteados en la Educación Secundaria Obligatoria son un ejemplo de formación integral, globalizadora, de formación de la persona. Yo es que no sé si hay más objetivos, consensuados en todo el país, con todas las comunidades autónomas, se tardó la tira en hacerlos, yo los leo y lo único que te pueden parecer es que son muchos, que son ambiciosos, pero si uno saca de ahí, eso es lo que todo el mundo quisiéramos de nuestros hijos. (MC Dir 231).

Pero se realizan en las sesiones de grupo algunas matizaciones acerca del sentido maximalista que suponen, o acerca de su generalidad. Pero en líneas generales hay que subrayar el nivel de acuerdo existente:

... en cuanto a los objetivos, que los tengo aqui, desde luego son muy generales. En nuestro Centro, bueno, pues ya es uno de los 
pioneros y ya hemos hecho la adaptación para los ciclos, pero desde luego son muy generales. Desde luego hay ucomprender y producir mensajes orales y escritos con propiedad, autonomía y creatividad". Esto en teoría está muy bien, pero es que al final de la etapa ¿cuántos alumnos lo consiguen? Y como éste que es el primero son los demás. (O Prof 182).

Las áreas de la Educación Secundaria Obligatoria, en una perspectiva general, alcanzan la valoración máxima en cuanto a la importancia que le conceden los profesores: supera los 4 puntos en una escala cuyo rango es de 1 a 5 . Sin embargo se percibe una situación conflictiva cuando entra en juego la carga horaria. La carga horaria atribuida a cada una de las áreas tiene una especial importancia. Entran en juego en esta cuestión intereses diversos, desde los atribuibles a la importancia y valoración de los contenidos de cada área para la formación de los alumnos hasta reivindicaciones corporativas. Pero apenas existe relación entre la importancia que se concede a cada área y el horario que se considera que hay que asignarle en la enseñanza. El horario que debería tener cada área y su valoración para la formación de los alumnos no son concordantes.

Las discrepancias entre valoración y horario parecen estar moduladas por la adscripción de los profesores a las áreas: los profesores de cada área tienden a demandar mayor número de horas para sus propias asignaturas:

... si vamos por gremios los músicos defienden la importancia de la música, y reivindican mayor carga lectiva, los tecnólogos lo mismo, y los de lengua lo mismo, con argumentos a favor de todas las reivindicaciones, pero algo hay que hacer y se haga lo que se haga.., por eso hablaba igual de pactar los previos desde dónde vamos a opinar, $y$ decir esta cantidad o reparto de carga horaria o por áreas o el número de áreas por curso nos parece el correcto o no estamos acertando, por esta razón o esta otra. (Nadir, 13).

Hay una relativa presencia de opiniones favorables a las "asignaturas tradicionales". Y de modo especial a las materias instrumentales:

... fallan mucho las áreas instrumentales .... La Lengua y las Matemáticas evidentemente quizá necesiten más horas, quizás, no sé, se tengan que dar de otra manera o lo que sea, pero lo que está clarísimo es que sin esas dos areas instrumentales todo lo demás, de alguna manera, se viene abajo. (LEProf, 38).

En estrecha relación con el problema de los horarios aparecen las actividades que se han determinado como alternativas a la enseñanza de la Religión. Estas actividades, de componente lúdico a veces, tienen en general dudosa consistencia. Y consumen un tiempo del horario que es significativo. 
La introducción dentro del horario de estas actividades genera discrepancias. Se resalta la incoherencia que supone el tiempo que ocupan cuando es tan escaso el dedicado a otras disciplinas que se consideran esenciales:

El dar tres horas en el ciclo de religión, o estudio dirigido como alternativa, como está puesto y con todo respeto a la gente, a los padres que optan por la religión, nos parece excesivo a mucha gente. Tres horas en el ciclo, teniendo en cuenta sobre todo que tenemos algunas asignaturas, como plástica y música, con una hora para cada curso y teniendo en cuenta también que el estudio dirigido se ha convertido en una hora en los Centros en los que se cuida a los crios para que hagan los deberes de casa. (VascProf 97).

La valoración de los temas transversales es positiva, y sus contenidos se aprecian de un modo especial:

$Y$ en cuanto a la trasversalidad, yo creo que es una cosa que está apartadísima, por lo menos en los Centros que yo conozco. Como filosofía está muy bien, ... pero a la hora de la verdad por la trasversalidad no sé da. Cuando podian ser temas de darle enganche porque son temas de actualidad y que, por lo tanto, están en la vida de esos adolescentes. Pero yo creo que se ha quedado ahí. (VascProf, 96).

Se considera frecuentemente que los temas transversales son de abordaje más fácil por los profesores de Primaria. Se percibe un cierto distanciamiento hacia ellos entre el profesorado procedente de Educación Secundaria:

Es mucho más fácil porque ya en la enseñanza media ahí juegan ya diferentes profesionales y tiene que haber un mutuo acuerdo entre ellos para dar, aunque sólo sea, pautas de conducta, o ideas clave. Me parece que la enseñanza media tiene una dificultad que es mucho más fácil de resolver en la enseñanza primaria. (SADir, 477).

Las materias optativas plantean también problemas peculiares en los Centros, como consecuencia de las posibilidades y las dificultades materiales de atender a la demanda. La casuística es muy amplia: desde las "optativas obligatorias" en los Centros que tienen una sola línea, hasta la oferta de asignaturas que pueden ser consideradas irrelevantes. De todos modos, y en el momento en el que se realiza el estudio de campo, se estima que la oferta es excesiva:

Es en la optatividad, o sea, la optatividad es muchisima, yo creo que habria que fijar unas optativas, y dentro de esas optativas, pues primar, ¿no?. Ya se prima en la Cultura Clásica, en cuanto a segunda Lengua; pero bueno, no que tengamos posibilidad de tener en un Centro trece o catorce optativas. (LEDir 135). 
Esta situación no es generalizable a momentos y contextos posteriores a la fecha de realización del trabajo de campo. Se percibe en el momento presente $-y$ de esta percepción no tengo constancia más que intuitiva- una reducción drástica de las materias optativas en algunas zonas.

\subsubsection{Los Proyectos Curriculares}

Estos objetivos y contenidos han de ser plasmados en algunos instrumentos de planificación de la tarea escolar. Cuadernos de preparación de lecciones, fichas, programaciones y proyectos curriculares son nombres que han ido recibiendo estos medios, que son muy similares, en una ceremonia que reviste en gran parte una dimensión retórica:

Bueno, eso tiene mucho que ver con lo que decía antes $[X X]$, de que los Proyectos Curriculares no se hicieron en el proceso que se debian de haber hecho, porque no hubo ni tiempo. O sea, al final se copiaron, hubo que hacer Proyectos Curriculares del segundo ciclo sin tener los del primero, hubo que hacer los Proyectos Curriculares sin tener los puestos educativos, sin un marco previo. (LE Dir 320).

El análisis que se ha efectuado pone de manifiesto la coexistencia de, al menos, tres modalidades de previsión inmediata del desarrollo curricular, y que se han identificado como:

a/. Planificación convencional, que supone la utilización de programaciones tanto individuales como de departamento, adaptaciones curriculares, considerando prioritariamente los objetivos que se pretendían conseguir con los alumnos, el material didáctico y los recursos disponibles. Supone una aceptación de una programación coherente y válida, pero con un cierto distanciamiento de los modos programadores propugnados por la reforma educativa:

Desde la Inspección, desde los equipos directivos, y también somos responsables los asesores de los C.P.R.s, nos lanzamos a poner en manos del profesorado toda una jerga y todo un conjunto de proyectos: el proyecto educativo de centro, el proyecto curricular de centro, el proyecto curricular de área, las programaciones, las unidades didácticas, los materiales, sin que todo aquello tuviese suficiente difusión, debate y contrastación con hacer vincular lo que ya se hacía con lo que se proponía, que no era radicalmente nuevo (ODir, 116).

b/. Programación experiencial, caracterizada por el uso de notas y apuntes personales del profesor, procedentes de cursos anteriores, el libro de texto y la guía didáctica que suele acompañarle: 
Pero no olvidemos que hay ciertos profesores que no han abierto las cajas rojas. Seamos realistas, que en el sistema también hay profesores y profesoras que desconocen ... Hay profesores que siguen con sus apuntes, con sus planteamientos anteriores, con sus libros anteriores. (VADir, 239).

cl. Programación basada en la Reforma, que supone la utilización de los instrumentos diseñados a partir de la reforma educativa: el proyecto educativo de Centro, el proyecto curricular y la programación anual:

Esas programaciones, todo ese proceso que va desde el proyecto educativo de Centro hasta las adaptaciones de aula, como proyectos curriculares, también, quiero decir, ¿son exactas?. Yo estoy convencido de ello, yo estoy convencido, hasta profesionalmente, de ello, como comprenderéis. (LEProf 235).

Los medios que componen estos tres tipos de programaciones han sido evaluados esencialmente a partir de dos cuestiones. En una se solicitaba que se expresara la importancia que, a juicio de los profesores, tenía cada uno de los medios de planificación. En la otra se pedía que se señalara la frecuencia de su utilización real en la enseñanza.

El Proyecto Educativo de Centro y el Proyecto Curricular se valoran de un modo discretamente positivo. Sin embargo se declara que su utilización es muy baja. Justamente lo contrario a lo que ocurre con instrumentos más convencionales y que se valoran sensiblemente menos que se utilizan. Las notas personales y, sobre todo, el libro de texto se valoran bajo, pero se utilizan mucho. Esta discrepancia, junto con las informaciones recogidas en los "grupos de diagnóstico", parece subrayar que el carácter burocrático y la disfuncionalidad alcanza a los medios que se proponen como alternativas, justo como en su momento ocurrió con otros.

El Proyecto Curricular hoy -como ayer fueron los Cuadernos de Preparación de Lecciones o las Programaciones Cortas y Largas- se ha convertido en una exigencia administrativa: se elabora, se envía al organismo correspondiente, se aprueba, y luego se ignora. No parece que estos instrumentos respondan a una necesidad sentida:

Eso es loable, pero es que ya están entregando, digamos pulcra y correctamente hechas y redactadas, las programaciones que dicen todo eso, pero en campo real no se hace caso. Eso, pues como tú has dicho, por lo menos en esta fase, está siendo una exigencia pues puramente burocrática, burocrática, como pasó en su día con otras. (SADir 317).

Sin embargo, y en una pregunta directa acerca de la necesidad de efectuar una programación o previsión del trabajo escolar, el profesorado reconoce su necesidad con una puntuación media de 4,6 sobre un recorrido de 1 a 5: 
(...) Bueno, yo aquí lo que tengo que decir es que no sé si porque a la fuerza ahorcan o porque realmente ha habido una, bueno que ha sido eficaz la formación que se ha impartido en los últimos tiempos, a mí me parece que hay cada vez más profesorado que prepara las clases, ya las preparaba antes evidentemente, pero que prepara las clases, precisamente, para adaptarse a toda una serie, no solamente de circunstancias de carácter pedagógico-didáctico, sino a circunstancias de carácter social. (...) (O Dir 179).

La instrumentación funcional y eficaz de la planificación de la enseñanza es un problema evidentemente no resuelto. El problema central estriba en apreciar cuáles son las metas de una reforma.

El problema de la previsión de la enseñanza, un problema de dimensión técnica con toda la serie de implicaciones ideológicas que se quiera, ha sido posiblemente instrumentalizada como categoría política.

\subsection{EL DESARROLLO CURRICULAR SEGÚN LOS PROFESORES DE ESO}

La descripción del diseño curricular, a partir de los medios utilizados en esta investigación, es mucho más fácil que la explicación de los modos de actuación de los profesores en el aula. Las distintas áreas, los materiales y recursos disponibles, los momentos evolutivos de los alumnos exigen, lógicamente, tratamientos y modos de intervención suficientemente diferenciados.

A ello es necesario sumar una cierta "cultura de la privacidad" desarrollada en torno a la actuación del profesor en el aula. Esa privacidad conlleva, a veces, la utilización del "veto de bolsillo": la inhibición por parte del profesor ante normas, principios o actividades que se recomienda utilizar en clase. (Boyd, 1987):

Yo te puedo decir que, en estos meses que han pasado, hoy por hoy no sé cuáles son las técnicas y las estrategias que mayoritariamente se utilizan en el centro. ¿Por qué? Porque no se habla. (VASCProf 329).

Seguramente habrá experiencias majísimas individuales. Por supuesto que sí, como ha habido hasta ahora y porque la práctica de alguna gente es fabulosa, aunque siempre podamos (...) Pero yo creo que son cosas a las que todavia no hemos llegado. Nos da miedo todavia hablar de ello. (VASCProf 330).

Y el uso de esa capacidad de "veto" no es otra cosa que un modo de plasmación operativa de la libertad de enseñanza. Lo más difícil de una reforma educativa -y uno de sus objetivos prioritarios- es modificar los modos de intervención en el aula. 
La más característica de las líneas de fuerza de las actividades didácticas es el constructivismo. Se pretendería, como principio básico, "facilitar la construcción de aprendizajes significativos, diseñando actividades de enseñanza y aprendizaje que permitan el establecimiento de relaciones sustantivas entre los conocimientos y experiencias previas y los nuevos aprendizajes":

Si como acaba de decir el que me antecede, es que uno de los problemas que tiene la ley ... es que introduce la metodología y no tenemos garantía de que esta metodología tenga la duración que tiene la ley, no sabemos si el constructivismo va a ser suplantado por otro tipo de metodología que yo personalmente tengo otras alternativas que me interesan más, pero ¿dónde esta mi libertad de cátedra? (VDir, 84).

Una segunda línea supone "propiciar una metodología activa por parte de los docentes", un planteamiento activo que "se concibe como un proceso de naturaleza fundamentalmente interna y no simplemente manipulativa":

Luego, con el tema de la metodología te pasa un poco lo mismo. Evidentemente que la metodología que había antes era una metodología expositiva, etc., ahora es mucho más dinámica, mucho más activa. Son actividades de aula, actividades de laboratorio. Y eso, ¿qué consecuencia tiene?, ¿negativa?

Pues la consecuencia que tiene es que las actividades te llevan tanto tiempo, su planificación, su preparación y tanto tiempo llevarlas a cabo con los chavales que, al final, realmente dices: "bueno, ¿qué contenidos, qué conceptos he dado yo durante todo el curso?". Al final se te queda prácticamente, de todos los contenidos que tenías que haber dado, se te queda, prácticamente, en la mitad. Dices: "bueno, entonces ¿qué estoy yo consiguiendo con todo esto?". (LEProf, 30 y 31).

El activismo exige una breve reflexión que puede arrojar luz sobre las reformas educativas de la enseñanza obligatoria en nuestro país. El activismo es el eje -según se declara reiteradamente en su introducción- de los Cuestionarios Nacionales para la Enseñanza Primaria de 1953. Todavía más: en el "Curso de Orientaciones Nacionales de la Enseñanza Primaria", que se celebró en Pamplona en junio de 1938, una de las ponencias de carácter didáctico propugna una enseñanza activa. El activismo sigue siendo el principio rector explícito o implícito de las reformas de 1965 y de 1970 . Y hoy, a sesenta años de aquella propuesta oficialista de activismo, se sigue pregonando la necesidad de una enseñanza activa. La única conclusión válida es que las reformas educativas, hasta ahora, no han conseguido la meta prevista en los procesos de enseñanza. No han sido eficaces. Este es un elemento más a considerar en la dificultad de modificar el desarrollo curricular, de "desarraigar" modos de actuación que se consideran obsoletos. Se habla en 
este sentido de resistencia al cambio, de inercia de los sistemas escolares, que tienen su máxima presencia en los aspectos metodológicos.

La tercera línea enlaza con la anterior en este último punto. Esta línea es la tendencia a la innovación. Se pretenden actitudes innovadoras en los procesos de enseñanza a fin de eliminar lo obsoleto. Pero el problema central es el criterio a seguir para declarar obsoleto un proceso, un recurso o un procedimiento de enseñanza:

Yo creo que el tema de la innovación educativa está totalmente olvidado $y$, sin embargo, yo creo que es el elemento dinámico. (VASCDir, 241).

En cierto momento se señaló la obsolescencia, por ejemplo, del libro de texto, como luego se verá Y la alternativa que se propuso se resolvió frecuentemente en la fotocopia de material impreso.

Con el señalamiento de estas contradicciones se pretende subrayar, sobre todo, las dificultades inherentes a la descripción de los modos de actuación del profesor. $\mathrm{Y}$ el conflicto que supone, en no pocos casos, su prescripción.

Sobre las dificultades que se acaban de poner de manifiesto, hay que tener en cuenta que la "calidad" de los datos sobre la actuación del profesor en clase es tal vez la menor de todos los aportados en esta investigación. No es fácil recomponer la actividad profesional en el aula a partir de la respuesta a la encuesta, ni son excesivas las aportaciones que se realizan a este apartado en las sesiones de los "grupos de diagnóstico". Otros procedimientos de recogida de información de carácter externo (observación, entrevistas individuales, observación del material generado por los alumnos ...) podrían arrojar informaciones de más calidad.

De todos modos, y teniendo en cuenta las limitaciones señaladas, cabría resumir en tres líneas dominantes los criterios metodológicos que se pretenden en la Educación Secundaria Obligatoria.

a/. Metodologia innovadora y participativa. Supone que el profesor desarrolla las clases con flexibilidad organizativa, frecuentemente en grupos, y utiliza una metodología activa y parcipativa que propicia debates e intercambio de opiniones. En gran parte coincide con la propuesta metodológica de la reforma:

Luego, con el tema de la metodología te pasa un poco lo mismo. Evidentemente que la metodología que había antes era una metodología expositiva, etc., ahora es mucho más dinámica, mucho más activa. Son actividades de aula, actividades de laboratorio. $Y$ eso, ¿qué consecuencia tiene?, ¿negativa? (...). (LEProf 30). 
b/. Metodología convencional. A través de ella se tiende a alternar, en el desarrollo de los temas, la explicación, la respuesta a las preguntas de los alumnos, el trabajo individual, las tareas para casa y su corrección en clase. La intención dominante es la de facilitar el aprendizaje de los alumnos:

Siempre hemos explicado, hemos preguntado, hemos intercalado preguntas, hemos hecho ejercicios, pero hemos explicado. $Y$ yo creo que las cosas van por ahí. Y hasta cierto punto, a pesar de la mala visión que tengo de la E.S.O. ¿no?, pues creo que el buen sentido y el buen juicio de los profesores va, sobre todo, en este aspecto de la metodologia, pues va a determinar que al final hagamos lo que nos parece oportuno y reconduzcamos un poco las cosas. (OProf193).

cl. Exposición magistral. Es la exposición de un tema de modo completo sin participación de los alumnos, que se limitan a tomar apuntes y realizar ejercicios:

$Y$, efectivamente, cuando, como yo veía cómo iban las cosas, pues hacia el mes y medio empecé a ver lo que sabian y no sabian absolutamente nada, pero absolutamente nada. Y entonces, claro, reduje el volumen de fotocopias, de papelitos, e hice lo que ahora mismo acaba de decir Carmen: "coger la tiza y empezar a explicar las cosas, que es la única forma que tenemos, con 30 alumnos, de hacer que aprendan». (OProf 191).

Estas tres metodologías, como ya ocurría con los modos de programación, coexisten en la Educación Secundaria Obligatoria, y con importancia relativamente similar.

\subsection{LOS MATERIALES CURRICULARES}

La valoración que los profesores hacen de los materiales y recursos didácticos ponen de manifiesto que los materiales de elaboración propia y los libros de texto son los más utilizados por los profesores, a bastante distancia de los restantes medios.

Si se tiene en cuenta que el material de elaboración propia, en la mayoría de los casos, tiene características asimilables al libro de texto -fotocopias, alguna transparencia obtenida de libros, apuntes, etc.- es necesario reconocer el papel dominante que ocupa el material verboicónico y, de modo especial, el libro de texto en la Educación Secundaria Obligatoria y en la enseñanza en general.

Estos resultados parecen apuntar hacia el hecho de que la polémica sobre el libro de texto se ha cerrado, al menos de momento, mediante una adopción y utilización masiva. Frente a algunas descalificaciones en la primera 
etapa de la reforma, su utilización se ha vuelto a recuperar y se ha generalizado su uso:

$Y$ entonces, de pronto se decidió que los libros de texto, aquello era malísimo, aquello era el demonio en el rincón del aula. Y entonces se sustituyeron por cientos y cientos de fotocopias, por cierto, de libros de texto. (TODir 280).

Esta generalización provoca una serie de problemas que se detectan en los "grupos de diagnóstico".

El Decreto de Currículo, merced a la perspectiva flexible y abierta con la que se concibe en Educación Secundaria Obligatoria, facilita la posibilidad de acercamiento a las áreas a través del desarrollo, para un mismo nivel, de contenidos diferentes.Pero cada proyecto editorial la orienta y modula de modo distinto. Esta orientación flexible se valora genéricamente, pero se señala que puede resultar disfuncional, por ejemplo, a efectos de traslados de alumnos. De hecho, el valor atribuido por los profesores al libro como pauta de programación -que ya se veía en el diseño curricular- supone un cierre del currículum, que teóricamente es abierto y flexible, y que únicamente puede entenderse como tal hasta que se decide el libro a utilizar:

(...) Además, las editoriales, haciendo una óptima politica editorial, hasta el punto de que no me he dado cuenta hasta un año después, han hecho unos curriculos muy cerrados: si escoges una editorial, estás listo, porque tienes que seguir con ella. Y esto es grave porque no siempre hay una uniformidad en la calidad. $Y$ además mezclan de una manera, quizás sí que lógica, pero no siempre universalmente aceptada, las distintas áreas disciplinares. (BProf 33).

El material didáctico, y de modo especial los libros de texto, dan lugar a una serie de esfuerzos familiares notables. Se habla incluso del volumen y peso -físico y económico- del material didáctico para cada curso:

Pero es que volvemos a las editoriales. Las editoriales han cogido $y$ han hecho un libro de Música asi porque lo tenían que vender mejor que la editorial vecina. $Y$ otro libro de Tecnología asi, $y$ otro libro de no sé qué así. Y vemos ya a los chiquillos con los carros. La L.O.G.S.E ha venido con el carro. La moda ha salido conjunta; el carrito y la L.O.G.S.E. Entonces dices: imadre mía lo que hemos avanzado! ipues si que hemos avanzado, todos sobre ruedas!... (ZProf 202).

De los restantes recursos, la prensa y los medios informáticos son los menos utilizados. Pese a que una y otro han tenido programas orientados a su difusión y su uso, parece que su resultado no ha sido el esperado. 
Por último, se consideran también parte del desarrollo curricular los usos evaluadores de los profesores.

La evaluación constituye un primer nivel en el que afloran las contradicciones entre planteamientos teóricos y dificultades prácticas. Y esta es la situación que se percibe en este trabajo.

Coexiste, con esa aceptación, un reconocimiento explícito de las dificultades formales que implica la evaluación.

Los problemas que se perciben por parte de los profesores son, esencialmente, de carácter didáctico y organizativo. Entre otros se pueden señalar los siguientes:

Se advierte global e intuitivamente una reducción de capacidades y un descenso del rendimiento de los alumnos, frente al nivel que era usual de la estructura anterior de la enseñanza:

El segundo aspecto se refiere al rendimiento. Quizá sea el más preocupante y es bastante general la opinión de que el rendimiento de los chavales ha bajado en relación a años anteriores, tanto si consideramos el Bachillerato, como la propia E.G.B. Oir que chicos y chicas cada vez estudian menos y saben menos es bastante frecuente, y en la mayor parte de los casos se culpa al sistema nuevo, a las familias a la propia sociedad que imita todo lo contrario de un trabajo serio por parte de los alumnos en la casa. (OProf 76).

Se denuncia igualmente la ausencia de precisión en las exigencias de evaluación del rendimiento, pese a la definición de criterios. Se acusa una falta de requisitos suficientemente acertados en los criterios de evaluación y de promoción de los alumnos. Se señala igualmente la necesidad de instrumentos accesibles y usuales para evaluar, y de un modo muy especial, las actitudes, normas y valores:

O sea, montones de profesores no tienen ni idea de lo que tienen que evaluar, asi de claro, si son conocimientos, si son capacidades, si son todo junto. ¿Cómo voy a ver yo la actitud?, pues yo no evalúo la actitud porque a mí no me da la gana evaluar la actitud, decía el otro día una (compañera). (MCDir 394).

\subsection{LA EVALUACIÓN Y LA PROMOCIÓN}

La aceptación teórica de los principios de la evaluación y la promoción en la Educación Secundaria Obligatoria -evaluación criterial, evaluación en equipo, consideración de conceptos, procedimientos y valores, funciones y sentido de la evaluación-cuentan con una aceptación global que hay que calificar como positiva por parte del profesorado. Sin embargo surge una 
serie de manifestaciones de descontento sobre los problemas que derivan de su aplicación a la realidad:

Sí, por imperativo legal o como la queramos llamar. Nosotros, los criterios de promoción aprobados por el Centro, por lo que veo, son bastante más duros que los aprobados por otros centros. Yo creo que hemos sido muy duros. Además este año los hemos modificado con mayor dureza todavía con respecto al año pasado (PMDir 314).

La promoción de un curso a otro es un tema que preocupa de modo muy especial a los profesores. Se acusa de ambigüedad en las normas con las que se orienta al profesor sobre los aspectos a considerar en la promoción. No resulta fácil convertir en elementos mínimamente operativos formulaciones como "alcanzar en términos globales los objetivos de un ciclo o curso":

Yo, yo lo que entiendo es que efectivamente es muy difícil de, de valorar o evaluar las capacidades, ieh!, entonces por eso, cuando se dice de que el alumno promociona con dos, o promociona con tres porque ha alcanzado las capacidades, yo la verdad, yo creo, lo veo muy difícil de llegar a una valoración real de que el alumno haya alcanzado realmente esas capacidades. Entonces, como yo soy de Ciencias, yo sé los nümeros, entonces ha aprobado, le queden tres o le queden cuatro. Pero eso de las capacidades yo me siento indefenso, yo no sé, no sé, tendría que tener cinco alumnos, jeh!, y entonces hacer una evaluación continua de esos cinco alumnos; pero yo lo veo complicado, yo de momento. (MUDir, 228).

Se señala la existencia de un fuerte rigorismo formalista existente en el proceso de evaluación, así como se indica la innecesaria exigencia de documentos múltiples y que se perciben como estrictamente burocráticos:

Si tú suspendes a 20, haces 20 informes personalizados, y ese informe pasa al tutor, al año que viene. El Director se encarga de repartirlos entre los profesores de área para que sepan a qué atenerse, o sea, por qué se les ha suspendido, porque a $2^{\circ}$ pasan por ley, que sepan cómo vienen, cómo van, en qué condiciones está, si ha promocionado por un motivo, por otro. O sea, es mucho papel, pero es un grado de consenso. (TOProf, 355).

La ampliación notable del número de documentos y de alumnos a evaluar. Los horarios de las áreas, al reducirse, han producido el efecto de ampliar el número de grupos de alumnos que ha de atender cada profesor para mantener las horas de docencia. Y, como consecuencia, se incrementa el número de evaluaciones:

Hay un exceso de información. Fijense que en todo esto, documentos de evaluación que traigo aquí, aqui el profesor se tendrá que 
dedicar a anotar una serie de datos, que los puede recabar directamente con controles orales, escritos o con lo que sea, referentes a consecución de objetivos, interés y esfuerzo, integración de los conceptos, utilización de los procedimientos, desarrollo de nuevas actitudes, etc., etc. Con tal diversidad de escalas, y de valoraciones, y además las valoraciones distintas, yo creo que el profesor, si se dedica a anotar estos datos de cada alumno, no tiene tiempo de enseñarles en la clase, ni tampoco tiempo de preparar trabajos originales y prácticos (...). (LEProf 75).

Se señalan asimismo las dificultades de coordinación entre los Institutos de Educación Secundaria y los Centros de Educación Primaria que imparten ESO:

Evaluación: Yo creo que falla la evaluación inicial; no la evaluación inicial en el propio Centro, sino creo que es fundamental una mayor coordinación entre Primaria y Secundaria, que eso está fallando. Hay coordinación porque se mandan informes, pero muy determinados y serios para los alumnos de integración, pero la mayoria de los alumnos el tutor hace más o menos fotocopias de una cosa, de unos impresos, pero no hay un informe detallado. El tutor de primero de Secundaria, o el tutor -hace un año cuando se adelantó tercero de Secundaria-, el tutor que recibia no conocía a esos alumnos, bien porque no había un informe detalladísimo de cada uno de los alumnos que venian a Secundaria, no había contacto con sus tutores; yo creo que eso sí, vía horarios o vía cómo, pero habría que fomentar muchisimo más la coordinación. Hay demasiada tradición, desde mi punto de vista, para los exámenes, en esto sí que ya casi todo el mundo está de acuerdo, que los exámenes son normales porque protestan los padres o tal, desde mi punto de vista no tiene ningún sentido y se desmonta rápidamente, luego lo haré si procede. (ZDir, 61).

Parece que la aceptación teórica de los planteamientos innovadores con relación a la evaluación no tienen un correlato claro en la modificación de los modos y técnicas de evaluar.

\section{A MODO DE CONCLUSIÓN}

De lo expuesto cabe señalar una serie de conclusiones, de las que habría que matizar algunas, y, en otros casos, profundizar más.

En primer lugar se percibe un notable nivel de aceptación de los planteamientos explícitos o implícitos en la Educación Secundaria Obligatoria. Las áreas, los contenidos, los objetivos, por ejemplo, son aceptados mayoritariamente por los profesores. 
Las quejas se manifiestan más en las vías de solución propuestas, en las respuestas de la Administración a las necesidades más notadas y señaladas. Algunos de los problemas que se acusan con mayor frecuencia son:

La necesidad de profundizar y precisar más en las vías para la realización de las previsiones de enseñanza, para efectuar las programaciones o los proyectos curriculares. Los profesores necesitan instrumentos válidos, no vías y procedimientos que se presenten como requisitos puramente burocráticos.

La reducción de burocracia en los procesos evaluadores, claramente cargados de una dimensión formalista más que de una función realmente diagnóstica.

La conveniencia de definir de otro modo los procedimientos de promoción de los alumnos. Los problemas derivan de la imprecisión de los criterios, así como de la promoción automática, por "imperativo legal", como señalan algunos.

Se percibe una presencia de uno de los fenómenos más frecuentemente detectados en cualquier cambio de actuación o de expectativas con relación a grupos sociales, sean profesionales o no: la inercia de los grandes grupos. Se siente la necesidad de cambiar los modos de actuar, pero no se lleva a cabo el cambio. Se valoran negativamente ciertos materiales curriculares, como el libro de texto, pero se utilizan con gran frecuencia.

Una valoración global de la reforma es difícil. Aparece toda una serie de tonos y de gamas en las respuestas que no cabría concluir las posturas que predominan. Tal vez sea dominante la postura de aceptación de los principios generales de la nueva legislación, pero existe una frecuente demanda de vías de solución, de búsqueda de recursos que no se les ha proporcionado. Parece que domina la valoración positiva sobre trabajo con nuevos modos. Diciéndolo en terminología de la reforma: se han cambiado las actitudes, no los procedimientos. 


\section{BIBLIOGRAFÍA}

BOYD, W.L.(1987). "Public Education Last Hurrah? Schizofrenia, Amnesia and Ingnorance in School Politics", in Educational Evaluation and Policy Analysis, vol. 9, n. 2, pp. 85-100.

MERTON, R.K. (1946). "The Focused Interview", en American Journal of Sociology, 51, p. 541.

MERTON, R.K.; FISKE, M. y KENDALL, P.I. (1956). The Focused Interview, Free Press, N. York.

Ministerio de Educación y Ciencia (1989). Diseño Curricular Base. Educación Primaria. Madrid.
RODRIGUEZ DIÉGUEZ, J.L. (1997). "La técnica de los Grupos de Diagnóstico como procedimiento para la evaluación de un currículum en cambio", en Profesorado. Revista de Curriculum y Formación de profesorado, n. 1, pp. 25-32.

RODRIGUEZ DIÉGUEZ, J.L. y otros (1998). Diagnóstico del Sistema Educativo. La escuela secundaria obligatoria 3. Planes de estudio y métodos de enseñanza. INCE, Ministerio de Educación y Cultura, Madrid.

\section{RESUMEN}

El objetivo de este estudio es revisar las contribuciones de los profesores de Educación Secundaria Obligatoria a su evaluación.

La base del trabajo son las sesiones de grupos de diagnóstico que se realizaron con un total de 72 horas de trabajo, con los que se realizó una base de datos de 6727 registros.

Se analizan las opiniones de los profesores sobre el diseño curricular, el desarrollo del currículum, los materiales curriculares y la evaluación.

Las principales conclusiones son las siguientes:

Los profesores aceptan mayoritariamente la reforma educativa.

Se produce una gran inercia con relación a los usos en la enseñanza.

Hay quejas con relación a las soluciones adoptadas.

Palabras clave: Reforma educativa, evaluación interna, grupos de interés, evaluación del currículum, sistema educativo. 


\section{ABSTRACT}

The objective of this study is to revise the contributions of the Secondary Education teachers to its evaluation.

The ground of work is constituted by sessions of diagnosis groups that were carried out with a total emmount of work of 72 hours. Information from this work was the source of a data base with 6727 records.

Teachers' opinions on curricular design, curriculum development, curricular materials and evaluation one analised.

The principal conclusions are the following:

The teachers largely accept the educational reform.

A great inertia is produced in relation to the uses in the teaching practice.

There are complaints in relation to the chosen solutions.

Key words: Interest groups, educative system, educativa reform, inner evaluation. 